University of Nebraska - Lincoln

DigitalCommons@University of Nebraska - Lincoln

September 1979

\title{
Photoionization of chalcogen and halogen atoms: Cross sections and angular distributions
}

\author{
Steven T. Manson \\ Georgia State University, Atlanta, Georgia \\ Alfred Msezane \\ Georgia State University, Atlanta, Georgia \\ Anthony F. Starace \\ University of Nebraska-Lincoln, astarace1@unl.edu \\ Siamak Shahabi \\ University of Nebraska - Lincoln
}

Follow this and additional works at: https://digitalcommons.unl.edu/physicsstarace

Part of the Physics Commons

Manson, Steven T.; Msezane, Alfred; Starace, Anthony F.; and Shahabi, Siamak, "Photoionization of chalcogen and halogen atoms: Cross sections and angular distributions" (1979). Anthony F. Starace Publications. 12.

https://digitalcommons.unl.edu/physicsstarace/12

This Article is brought to you for free and open access by the Research Papers in Physics and Astronomy at DigitalCommons@University of Nebraska - Lincoln. It has been accepted for inclusion in Anthony F. Starace Publications by an authorized administrator of DigitalCommons@University of Nebraska - Lincoln. 


\title{
Photoionization of chalcogen and halogen atoms: Cross sections and angular distributions
}

\author{
Steven T. Manson and Alfred Msezane* \\ Department of Physics, Georgia State University, Atlanta, Georgia 30303
}

Anthony F. Starace and Siamak Shahabi

Behlen Laboratory of Physics, University of Nebraska, Lincoln, Nebraska 68588

(Received 29 March 1979)

\begin{abstract}
The authors present a survey of the photoionization cross sections and photoelectron angular distributions for the outer $p$ subshell of the chalcogen atoms $\mathrm{O}, \mathrm{S}, \mathrm{Se}$, and $\mathrm{Te}$ and the halogen atoms $\mathrm{F}, \mathrm{Cl}, \mathrm{Br}$, and $\mathrm{I}$. Results are presented in the Hartree-Fock approximation as well as in the central-potential-model approximation. An extensive analysis and comparison of the results obtained is presented which finds that exchange-interaction effects are strongest for the $n=3$ row elements.
\end{abstract}

\section{INTRODUCTION}

The theory of atomic photoionization has progressed rapidly since the late 1960 s in response to newly available experimental measurements in the vacuum-ultraviolet wavelength range. ${ }^{1-4}$ For the most part, however, both experiment and theory have concentrated on the study of closedshell atoms since these are the easiest to produce in the form of monatomic vapors in the laboratory and since the spherical symmetry of closed-shell systems simplifies the theoretical effort required. It remains true today that the interaction of radiation with approximately $75 \%$ of the elements of the Periodic Table is unstudied experimentally. ${ }^{2,5}$

Theoretical studies for these same elements have with few exceptions been limited to model potential calculation ${ }^{1,3,6}$ for the integrated photoionization cross sections. Such calculations account for the effects of the term level structure of open-shell atoms on the cross sections only to the extent of incorporating the differing binding energies of each ionic term level in the calculations.

In this paper we present theoretical calculations of the photoionization cross sections and photoelectron angular distributions of the outer $p$ subshell of the chalcogen atoms $\mathrm{O}, \mathrm{S}$, Se, and Te and the halogen atoms $\mathrm{F}, \mathrm{Cl}, \mathrm{Br}$, and $\mathrm{I}$. Our purpose is to provide the same theoretical understanding for the outer-shell photoabsorption spectrum of open-shell atoms having outer configurations $p^{4}$ and $p^{5}$ as has been provided in an earlier pape ${ }^{7}$ for the closed-shell atoms having outer configuration $p^{6}$. All our calculations employ Hartree-Fock continuum orbitals calculated in the field of a relaxed ionic core. Dynamical effects of atomic and ionic term level structure are thus explicitly taken into account and comparison is made with model potential calculations that ignore such term level effects. On the other hand, our results should be regarded only as the best possible zeroth-order results since we have ignored coupling between final-state photoelectron channels and configuration interaction effects in both the initial atomic state and the final ionic state. These effects of electron correlation that we have ignored may be expected to change our results quantitatively, but should not affect the qualitative features that it is our purpose to survey.

Other theoretical work for the chalcogens and halogens-as indeed for most open-shell atomsis rather sparse. Model potential calculations of the photoionization cross sections for all the elements considered in this paper have been carried out by McGuire. ${ }^{8}$ A number of more recent model potential calculations have been carried out for the photoionization cross section of atomic oxygen..$^{9-12}$ Manson has used a model potential to survey the photo-electron angular distribution from $p$ subshells for selected elements throughout the Periodic Table; while the chalcogens and halogens were not studied, Manson found certain features of the photoelectron angular distribution that are common to all $p$ subshells. ${ }^{13}$ More advanced theoretical calculations (i.e., at the level of the Hartree-Fock approximation or better) for photoionization cross sections and angular distributions of the chalcogen and halogen atoms have only been done for atomic oxygen, ${ }^{14-20}$ sulfur, ${ }^{21-23}$ and chlorine. ${ }^{24-27}$

\section{THEORETICAL SUMMARY}

Consider the general electric dipole photoionization process in $L S$ coupling involving the outer $p$ subshell of an atom $A$ :

$$
A n p^{\alpha}\left(L_{0} S_{0}\right)+\omega \rightarrow A^{+} n p^{\alpha-1}\left(L_{c} S_{c}\right) \in l\left(L S_{0}\right) .
$$

The orbital and spin angular momenta of the atom and of the ion core have been denoted by $L_{0} S_{0}$ and $L_{c} S_{c}$, respectively. Electric dipole selection rules 
restrict the photoelectron orbital angular momentum $l$ to the values 0 and 2 (in the single-configuration approximation) and the final-state orbital angular momentum $L$ to the values $L_{0}, L_{0} \pm 1$. The occupation number of the open $p$ subshell is indicated by $q$. Atomic units will be used throughout this section, i.e., $m=e=\hbar=1$.

A. Formula for the photoionization cross section

In the approximation that one ignores interaction between alternative final-state channels, ${ }^{28}$ the photoionization cross section for leaving the ion in the term level $L_{c} S_{c}$, i.e., for the process

$$
A n p^{q}\left(L_{0} S_{0}\right)+\omega \rightarrow A^{+} n p^{q-1}\left(L_{c} S_{c}\right)+e^{-}
$$

is given by summing the cross section for reaction (1) over the final state quantum numbers $l$ and $L$ (Ref. 1):

$$
\sigma\left(L_{c} S_{c}\right)=\left.\frac{4 \pi^{2} \omega}{3 c\left[L_{0}\right]} \sum_{i, L}\left\langle A n p^{\alpha} L_{0} S_{0}\left|\sum_{i=1}^{N} r_{i}^{(1)}\right| A^{+} n p^{\alpha-1}\left(L_{c} S_{c}\right) \in l L S_{0}\right\rangle\right|^{2} .
$$

In Eq. (3a) the symbol $\left[L_{0}\right]$ indicates $2 L_{0}+1$ and the reduced matrix element of the electric dipole tensor operator is given by

$$
\begin{aligned}
\left\langle A n p^{\alpha} L_{0} S_{0}\left|\sum_{i=1}^{N} r_{i}^{(1)}\right| A^{+} n p^{\alpha-1}\left(L_{c} S_{c}\right) \in l L S_{0}\right\rangle & =q^{1 / 2}\left(n p^{\alpha} L_{0} S_{0}\left\{\mid n p^{\alpha-1} L_{c} S_{c}\right)\left\{S_{0} S_{c} \frac{1}{2}\right\}(-1)^{1+L_{c}+L_{0}}\right. \\
& \times\left[L_{0}\right]^{1 / 2}[L]^{1 / 2}\left\{\begin{array}{ccc}
1 & L_{0} & L_{c} \\
L & l & 1
\end{array}\right\}\left\langle n p \| r^{(1)}|| \epsilon l\right\rangle,
\end{aligned}
$$

where

$$
\begin{aligned}
& \left\langle n p|| r^{(1)}|| \epsilon l\right\rangle \\
& \quad\left\{\begin{array}{l}
-\sqrt{2} \int_{0}^{\infty} P_{n p}^{L_{0} S_{0}}(r) r P_{\epsilon d}^{L_{c} S_{c} L}(r) d r \text { for } l=2 \\
+\int_{0}^{\infty} P_{n p}^{L_{0} S_{0}}(r) r P_{\epsilon s}^{L_{c} S_{c} L}(r) d r \text { for } l=0 .
\end{array}\right.
\end{aligned}
$$

In Eq. (3b) the first set of parentheses indicate a coefficient of fractional parentage, ${ }^{29}$ the first set of curly brackets indicate a triangular $\delta$ function, ${ }^{30}$ and the second set of curly brackets indicate a $6-j$ symbol. ${ }^{31}$ In Eq. (3c) the radial wave functions of the photoelectron are indicated by $r^{-1} P_{n p}^{L_{0} S_{0}}(r)$ for the initial state and by $r^{-1} P_{6 l}^{L_{C} S_{c} L}(r)$ for the final state. The superscripts indicate that these orbitals are term dependent.

\section{B. Formulas for the photoelectron angular distribution}

The differential cross section for reaction (2) may be written quite generally (in the electric dipole approximation) $\mathrm{as}^{32}$

$$
\frac{d \sigma\left(L_{c} S_{c}\right)}{d \Omega}=\frac{\sigma\left(L_{c} S_{c}\right)}{4 \pi}\left[1+\beta\left(L_{c} S_{c}\right) P_{2}(\cos \theta)\right]
$$

Equation (4) assumes that the incident light is linearly polarized, that the target is unpolarized, and that $\theta$ is measured with respect to the polarization direction. $P_{2}(\cos \theta)$ is a Legendre polynomial and $\beta\left(L_{c} S_{c}\right)$ is the asymmetry parameter, whose values are limited to the range

$$
-1 \leqslant \beta \leqslant 2
$$

by the requirement that the differential cross section, Eq. (4), is always non-negative. The dynamical information of the angular distribution is contained in the energy-dependent asymmetry parameter. [Note that although Eq. (4) assumes the incident light is linearly polarized, formulas for the differential cross section for any kind of light polarization may be written in terms of the same asymmetry parameter $\left.\beta\left(L_{c} S_{c}\right){ }^{1,3}\right]$

Although formulas for the angular distribution asymmetry parameter in $L S$ coupling have been given by Lipsky ${ }^{33}$ and by Jacobs and Burke, ${ }^{34,35}$ we have used the angular-momentum-transfer formulations for $\beta$ of Dill and $\mathrm{Fano}^{36-38}$ for two reasons. First, this latter formulation represents $\beta$ as an incoherent sum of contributions characterized by a fixed value of the angular momentum transfer $j_{t}$, which is defined below. This decomposition simplifies the theoretical analysis. Second, this formulation permits a transparent reduction, within certain approximations, to the simpler formulation for $\beta$ of Cooper and Zare. ${ }^{39}$

The angular momentum transfer $j_{t}$ for reaction (1) above is defined in $L S$ coupling by

$$
\overrightarrow{\mathrm{j}}_{t}=\overrightarrow{\mathrm{j}}_{p}-\overrightarrow{\mathrm{I}}=\overrightarrow{\mathrm{L}}_{c}-\overrightarrow{\mathrm{L}}_{0}
$$

where $j_{p}$ is the angular momentum of the incident photon (which is equal to unity in the electric dipole approximation). Thus, in $L S$ coupling, $j_{t}$ equals the orbital angular momentum transfer red between 
the atom and the ion core. The allowed values of $j_{t}$ are given by Eq. (6) subject to the restrictions imposed by angular momentum conservation, i.e.,

$$
\overrightarrow{\mathrm{L}}_{0}+\overrightarrow{\mathrm{j}}_{p}=\overrightarrow{\mathrm{L}}_{c}+\overrightarrow{\mathrm{I}}
$$

and by parity conservation, i.e.,

$$
\pi_{0} \pi_{p}=\pi_{c} \pi_{e} \text { or } \pi_{0}(-1)=\pi_{c}(-1)^{l},
$$

where the second expression uses the fact that the parity of the photon is $\pi_{p}=-1$ and that the parity of the photoelectron is $\pi_{e}=(-1)^{l}$.

The asymmetry parameter $\beta$ appropriate for substitution in Eq. (4) is given by the following weighted average,

$$
\beta=\frac{\sum_{j_{t}} \sigma\left(j_{t}\right) \beta\left(j_{t}\right)}{\sum_{j_{t}} \sigma\left(j_{t}\right)}
$$

where all quantities in Eq. (9) depend implicitly on the state of the ion core (i.e., on $L_{c} S_{c}$ ). The sum in Eq. (9) extends over all allowed values of $j_{t} . \quad \beta\left(j_{t}\right)$ and $\sigma\left(j_{t}\right)$ are the asymmetry parameter and the partial cross section characteristic of a given value of $j_{t}$. They are defined in terms of scattering amplitudes $S_{l}\left(j_{t}\right)$ as follows:

$$
\beta_{\text {far }}\left(j_{t}\right)=\frac{\left(j_{t}+2\right)\left|S_{+}\left(j_{t}\right)\right|^{2}+\left(j_{t}-1\right)\left|S_{-}\left(j_{t}\right)\right|^{2}-3\left[j_{t}\left(j_{t}+1\right)\right]^{1 / 2}\left\{S_{+}\left(j_{t}\right) S_{-}^{\dagger}\left(j_{t}\right)+\text { c.c. }\right\}}{\left(2 j_{t}+1\right)\left\{\left|S_{+}\left(j_{t}\right)\right|^{2}+\left|S_{-}\left(j_{t}\right)\right|^{2}\right.},
$$

$$
\begin{aligned}
& \beta_{\text {unf }}\left(j_{t}\right)=-1, \\
& \sigma_{\text {fav }}\left(j_{t}\right) \propto\left(2 j_{t}+1\right)\left\{\left|S_{+}\left(j_{t}\right)\right|^{2}+\left|S_{-}\left(j_{t}\right)\right|^{2}\right\}, \\
& \sigma_{\text {unf }}\left(j_{t}\right) \propto\left(2 j_{t}+1\right)\left|S_{0}\left(j_{t}\right)\right|^{2} .
\end{aligned}
$$

In Eq. (10) the subscripts "fav" and "unf" refer to whether the value of $j_{t}$ is "parity favored" or "parity unfavored," according to the following scheme $^{37}$ :

$$
(-1)^{j} t=\pi_{0} \pi_{c} \Rightarrow j_{t} \text { is parity favored, }
$$

$$
(-1)^{j} t=-\pi_{0} \pi_{c} \Rightarrow j_{t} \text { is parity unfavored. }
$$

Also, in Eq. (10) the subscripts “+”, “-”, and " 0 " on the amplitudes $S_{l}\left(j_{t}\right)$ denote whether $l=j_{t} \pm 1$ or $l=j_{t}$, respectively; "c.c." denotes "complex conjugate"; and the proportionality constant in Eqs. (10c) and (10d) has been omitted since it cancels in Eq. (9).

The scattering amplitudes $S_{l}\left(j_{t}\right)$ have been obtained in $L S$ coupling in the approximation that final-state interchannel coupling is ignored by Dill, Manson, and Starace ${ }^{22,23}$ as

$$
\begin{aligned}
S_{l}\left(j_{t}\right) \propto i^{-l} \exp \left(i \sigma_{\epsilon l}\right)[l]^{1 / 2}\left(\begin{array}{lll}
l & 1 & l_{0} \\
0 & 0 & 0
\end{array}\right) \\
\times \sum_{L} \exp \left(i \delta_{\epsilon l}^{L_{c} S_{c} L}\right) R_{\epsilon l}^{L_{c} S_{c} L}[L] \\
\times\left\{\begin{array}{ccc}
L_{0} & L_{c} & j_{t} \\
l & 1 & L
\end{array}\right\}\left\{\begin{array}{lll}
L_{0} & L_{c} & l_{0} \\
l & 1 & L
\end{array}\right\} .
\end{aligned}
$$

In Eq. (12) $l_{0}$ is the photoelectron's initial orbital angular momentum (which is unity for $p$ subshells),
$\delta_{\epsilon}^{L} \mathcal{G}_{c} S^{L}$ is the photoelectron's phase shift with respect to a Coulomb wave, $\sigma_{\epsilon l}$ is the Coulomb phase shift [i.e., $\arg \Gamma(l+1-i / k)$, where $\epsilon($ a.u. $\left.)=\frac{1}{2} k^{2}\right]$, and $R_{\epsilon l}^{L_{C} S_{c} L}$ is the radial dipole matrix integral,

$$
R_{\epsilon i}^{L_{c} S_{c} L} \equiv \int_{0}^{\infty} P_{n l_{0}}^{L_{0} S_{0} L}(r) r P_{\epsilon i}^{L_{c} S_{c} L}(r) d r .
$$

The constant of proportionality is omitted in Eq. (12) since it cancels in Eq. (9). Equations (9)-(12) are thus the formulas used in this paper to calculate the asymmetry parameter $\beta$.

It is of interest to see how the angular-momentum-transfer formulation for $\beta$ reduces to the simpler expression of Cooper and Zare. ${ }^{39}$ There are two ways in which this reduction takes place. The first is an approximate reduction which holds for those atoms in which the electron-ion interaction may be said to be isotropic, i.e., the photoelectron phase shifts and the radial dipole matrix elements become approximately independent of the term levels:

$$
\exp \left(i \delta_{\epsilon l}^{L_{c} S_{c} L}\right) R_{\epsilon l}^{L_{c} S_{c} L} \underset{\begin{array}{c}
\text { 1sotropic } \\
\text { Interactions }
\end{array}}{\longrightarrow} \exp \left(i \delta_{\epsilon l}\right) R_{\epsilon l} .
$$

Substituting Eq. (14) in Eq. (12) and summing over $L$ gives

$$
\begin{aligned}
S_{l}\left(j_{t}\right) \propto & \delta\left(j_{t}, l_{0}\right) i^{-l} \exp \left(i \sigma_{\epsilon l}\right)[l]^{1 / 2} \\
& \times\left(\begin{array}{lll}
l & 1 & l_{0} \\
0 & 0 & 0
\end{array}\right) \exp \left(i \delta_{\epsilon l}\right) R_{\epsilon l} .
\end{aligned}
$$

Note that in Eq. (15) the $\delta$ function indicates that only the single value $j_{t}=l_{0}$ is permitted. Substitution of Eq. (15) in Eq. (9) then gives the Cooper-Zare expression for $\beta$ (with subscripts $\epsilon$ omitted $)^{39}$ : 


$$
\beta^{\mathrm{CZ}}=\frac{l_{0}\left(l_{0}-1\right) R_{l_{0-1}}^{2}+\left(l_{0}+1\right)\left(l_{0}+2\right) R_{l_{0+1}}^{2}-6 l_{0}\left(l_{0}+1\right) R_{l_{0-1}} R_{l_{0+1}} \cos \left(\sigma_{l_{0}+1}+\delta_{l_{0+1}}-\sigma_{l_{0-1}}-\delta_{l_{0-1}}\right)}{\left(2 l_{0}+1\right)\left[l_{0} R_{l_{0}-1}^{2}+\left(l_{0}+1\right) R_{l_{0}+1}^{2}\right]} .
$$

The second way in which Eq. (9) reduces to Eq. (16) is an exact reduction. It occurs when $L_{0}=0$, in which case Eq. (12) once again reduces to Eq. (15) and only a single value of $j_{t}$ is allowed. For the chalcogen and halogen atoms studied in this paper $L_{0}=1$ for the atomic ground state. Hence, to the extent that the photoelectron-ion interactions are anisotropic, the reduction in Eq. (14) will not apply and we shall expect (a) that for a given ioncore term level $L_{c} S_{c}$, the $\beta$ parameter in Eq. (9) will have contributions from values of $j_{t} \neq l_{0}$, and (b) that the $\beta$ parameters calculated according to Eq. (9) will differ for different ionic term levels $L_{c} S_{c}$.

For the chalcogens and halogens considered in this paper, the geometrical factors which determine the photoelectron angular distribution are the same in the $L S$-coupling approximation. This identity stems from the fact that both groups of elements have the same initial orbital angular momentum $L_{0}$ (i.e., the ground-state term is ${ }^{3} P$ for the chalcogens and ${ }^{2} P$ for the halogens), the same values of ionic-core orbital angular momentum (i.e., the ionic term levels are ${ }^{4} S,{ }^{2} P$, and ${ }^{2} D$ for the chalcogens and ${ }^{1} S,{ }^{3} P$, and ${ }^{1} D$ for the halogens), and the same values of final orbital angular momentum $L$ (i.e., the final-state term levels are ${ }^{3} S,{ }^{3} P$, and ${ }^{3} D$ for the chalcogens and ${ }^{2} S,{ }^{2} P$, and ${ }^{2} D$ for the halogens). Hence from Eq. (6) the allowed values of $j_{t}$ are the same, and from Eq. (12) the geometrical factors which determine $S_{l}\left(j_{t}\right)$ are the same. Thus the dependence of the asymmetry parameter $\beta$ on the phase shifts and radial dipole matrix elements is the same for the chalcogens and the halogens, as may be seen from Eqs. (9) and (10).

\section{Numerical details}

Hartree-Fock (HF) wave functions with full allowance for exchange were used in these calculations. The discrete ground-state wave functions for each of the atoms were obtained from the tabulation of HF wave functions of Clementi and Roetti. ${ }^{40}$ The final continuum-state wave functions were obtained with full relaxation of the residual ion core. The HF wave functions for these ions were obtained for $\mathrm{O}^{+}, \mathrm{F}^{+}, \mathrm{S}^{+}, \mathrm{Cl}^{+}, \mathrm{Se}^{+}$, and $\mathrm{Br}^{+}$ from the above tabulation ${ }^{40}$ and for $\mathrm{Te}^{+}$and $\mathrm{I}^{+}$by running the Clementi computer code. ${ }^{41}$ Then, with the ionic orbitals fixed, each continuum oneelectron HF orbital for the photoelectron was calculated in the field of the fully relaxed orbitals of a given positive ion.

Specifically, for photoionization of an outer $p$ electron from one of the halogen or chalcogen atoms, the $\mathrm{HF}$ equation for $(n p)^{q} \rightarrow(n p)^{q-1} \in S$ is

$$
\begin{gathered}
\left(\frac{d^{2}}{d r^{2}}+\frac{2}{r} U(r)+\epsilon\right) P_{\epsilon s}(r)+\sum_{n^{\prime}=1}^{n}\left(\frac{2}{r} Y^{0}\left(n^{\prime} s, \epsilon s\right)-\lambda_{n^{\prime} s}\right) P_{n^{\prime} s}(r)+\frac{2}{r} \sum_{n^{\prime}=2}^{n-1} Y^{1}\left(n^{\prime} p, \epsilon s\right) P_{n^{\prime} p}(r) \\
+\frac{2}{r} \sum_{n^{\prime}=3}^{n-1} Y^{2}\left(n^{\prime} d, \epsilon s\right) P_{n^{\prime} d}(r)+\frac{2}{r} \frac{3 q-3-b^{\prime}}{18} Y^{1}(n p, \epsilon s) P_{n p}(r)=0
\end{gathered}
$$

and the HF equation for $(n p)^{\alpha} \rightarrow(n p)^{\alpha-1} \in d$ is

$$
\begin{aligned}
& {\left[\frac{d^{2}}{d r^{2}}-\frac{6}{r^{2}}+\frac{2}{r}\left(U(r)-\frac{a^{\prime \prime}}{105} Y^{2}(n p, n p)\right)\right] P_{\epsilon d}(r)+\frac{2}{5 r} \sum_{n^{\prime}=1}^{n} Y^{2}\left(n^{\prime} s, \epsilon d\right) P_{n^{\prime} s}(r)} \\
& +\frac{2}{r} \sum_{n^{\prime}=2}^{n-1}\left[\frac{2}{5} Y^{1}\left(n^{\prime} p, \epsilon d\right)+\frac{9}{35} Y^{3}\left(n^{\prime} p, \epsilon d\right)\right] P_{n^{\prime} p}(r) \\
& +\sum_{n^{\prime}=3}^{n-1}\left(\frac{2}{r}\left[Y^{0}\left(n^{\prime} d, \epsilon d\right)+\frac{2}{7} Y^{2}\left(n^{\prime} d, \epsilon d\right)+\frac{2}{7} Y^{4}\left(n^{\prime} d, \epsilon d\right)\right]-\lambda_{n^{\prime} d}\right) P_{n^{\prime} d}(r) \\
& +\frac{2}{r}\left(\frac{6 q-6-b^{\prime \prime}}{90} Y^{1}(n p, \epsilon d)+\frac{21 q-21-c^{\prime \prime}}{490} Y^{3}(n p, \epsilon d)\right) P_{n p}(r)=0 .
\end{aligned}
$$

In these continuum HF equations, we have defined the potential $U(r)$ as

$$
\begin{aligned}
U(r)=Z & -2 \sum_{n^{\prime}=1}^{n} Y^{0}\left(n^{\prime} s, n^{\prime} s\right)-6 \sum_{n^{\prime}=2}^{n-1} Y^{0}\left(n^{\prime} p, n^{\prime} p\right) \\
& -10 \sum_{n^{\prime}=3}^{n-1} Y^{0}\left(n^{\prime} d, n^{\prime} d\right)-(q-1) Y^{0}(n p, n p),
\end{aligned}
$$

where the symbols $Y^{\lambda}$ in Eqs. (17) and (18) stand for the following integrals:

$$
\begin{aligned}
\frac{1}{r} Y^{\lambda}\left(n l, \epsilon l^{\prime}\right) & =\frac{1}{r^{\lambda+1}} \int_{0}^{r} r^{\prime \lambda} P_{n l}\left(r^{\prime}\right) P_{\epsilon l^{\prime}}\left(r^{\prime}\right) d r^{\prime} \\
& +r^{\lambda} \int_{r}^{\infty} \frac{P_{n l}\left(r^{\prime}\right) P_{\epsilon l^{\prime}}\left(r^{\prime}\right)}{r^{\lambda+1}} d r^{\prime}
\end{aligned}
$$


TABLE I. Allowed transitions for photoionization of the outer $p$ subshell of the halogen and chalcogen atoms within the single-configuration approximation.

\begin{tabular}{|c|c|c|c|}
\hline Initial state & $\begin{array}{c}\text { Final-ionic-state } \\
\text { term level }\end{array}$ & $\begin{array}{l}\text { Halogens } \\
\text { Photoelectron } \\
\text { state }\end{array}$ & $\begin{array}{l}\text { Final-state } \\
\text { term level }\end{array}$ \\
\hline$\left(n p^{5}\right)^{2} P$ & $\begin{array}{r}\left(n p^{4}\right)^{1} S \\
{ }^{3} P \\
{ }^{1} D\end{array}$ & $\begin{array}{l}\epsilon_{S} \\
\epsilon_{d} \\
\epsilon_{s} \\
\epsilon_{d} \\
\epsilon_{s} \\
\epsilon_{d}\end{array}$ & $\begin{array}{l}{ }^{2} S \\
{ }^{2} D \\
{ }^{2} P \\
{ }^{2} P,{ }^{2} D \\
{ }^{2} D \\
{ }^{2} S,{ }^{2} P,{ }^{2} D\end{array}$ \\
\hline$\left(n p^{4}\right)^{3} P$ & $\begin{array}{r}\left(n p^{3}\right)^{4} S \\
{ }^{2} P \\
{ }^{2} D\end{array}$ & $\begin{array}{c}\text { Chalcogens } \\
\qquad \epsilon_{S} \\
\epsilon_{d} \\
\epsilon_{s} \\
\epsilon_{d} \\
\epsilon_{s} \\
\epsilon_{d}\end{array}$ & $\begin{array}{l}{ }^{3} S \\
{ }^{3} D \\
{ }^{3} P \\
{ }^{3} P,{ }^{3} D \\
{ }^{3} D \\
{ }^{3} S,{ }^{3} P,{ }^{3} D\end{array}$ \\
\hline
\end{tabular}

in which the bound orbitals are those for the ion. $\epsilon$ is the photoelectron energy (in rydbergs, 13.6 $\mathrm{eV}), \lambda_{n^{\prime} s}$ and $\lambda_{n^{\prime} d}$ are the off-diagonal parameters to ensure orthogonality of the continuum function to the ionic orbitals of the same symmetry, and the $b^{\prime}, a^{\prime \prime}, b^{\prime \prime}$, and $c^{\prime \prime}$ are all term dependent and are given elsewhere. ${ }^{42}$ Thus, although for the sake of clarity the term dependence of the orbitals has been suppressed, this dependence is nonetheless there. The interaction matrix elements needed to derive these equations are given by Slater. ${ }^{43}$

The equations were solved iteratively, using a Hartree-Slater ${ }^{44}$ (HS) continuum wave function as the starting point. The off-diagonal parameters were calculated according to

$$
\lambda_{n^{\prime} s}=-\frac{1}{18} b^{\prime}\left\langle n^{\prime} s\left|(2 / r) Y^{1}(n p, \epsilon s)\right| n p\right\rangle
$$

and

$$
\begin{aligned}
\lambda_{n^{\prime} d}=-\frac{1}{105} a^{\prime \prime}\left\langle n^{\prime} d\right| & (2 / r) Y^{2}(n p, n p)|\epsilon d\rangle \\
-\left\langle n^{\prime} d\right|(2 / r) & {\left[\frac{1}{90} b^{\prime \prime} Y^{1}(n p, \epsilon d)\right.} \\
& \left.+\frac{1}{490} c^{\prime \prime} Y^{3}(n p, \epsilon d)\right]|n p\rangle
\end{aligned}
$$

which may be obtained by comparing Eqs. (17) for the continuum orbitals with the corresponding $\mathrm{HF}$ equations for the ionic orbitals. ${ }^{7,14}$

The calculations were carried out for each of the nine allowed transitions, shown in Table I, for each of the four halogen and chalcogen atoms. Note that this table shows the transitions which give a nonzero contribution to the cross section using single-configuration $\mathrm{HF}$ wave functions for the initial and final states. Angular momentum and parity considerations alone allow other transitions which would contribute if configuration in- teraction in either the initial or final state is taken into account. For example, for the halogens the photoionizing transition

$$
\left(n p^{5}\right)^{2} P+\omega \rightarrow\left[\left(n p^{4}\right)^{1} D \epsilon g\right]^{2} D
$$

satisfies all the selection rules but has zero amplitude in the single configuration approximation that we have adopted.

The dipole matrix elements, Eq. (13), were calculated in both the "length" and "velocity" approximation $s^{1,3,7}$ but only the results based upon the "length" approximation using experimental ionization potentials are presented herein. This choice has been made because it is the "length" form of the dipole matrix element which is gauge invariant using a HF Hamiltonian ${ }^{45,46}$ as well as the fact that experience has shown that the "length" formula is generally superior (closer to experiment) for HF oscillator-strength calculations. ${ }^{7,47}$ Note however that the photoelectron angular distribution asymmetry parameter $\beta$ has been shown to be generally rather insensitive to the dipole matrix elements and hence to the formula used to calculate these matrix elements; the phase shifts are far more crucial determinants of $\beta .^{7,48}$

\section{RESULTS AND DISCUSSION}

\section{A. Cross sections}

The calculated cross sections for outer $p$ subshells of the halogens ( $\mathrm{F}, \mathrm{Cl}, \mathrm{Br}, \mathrm{I})$ and the chalcogens $(\mathrm{O}, \mathrm{S}, \mathrm{Se}, \mathrm{Te})$ are given in Figs. 1 and 2 , respectively. For each element, we present the total cross section as well as the partial cross sections, which correspond to particular term 

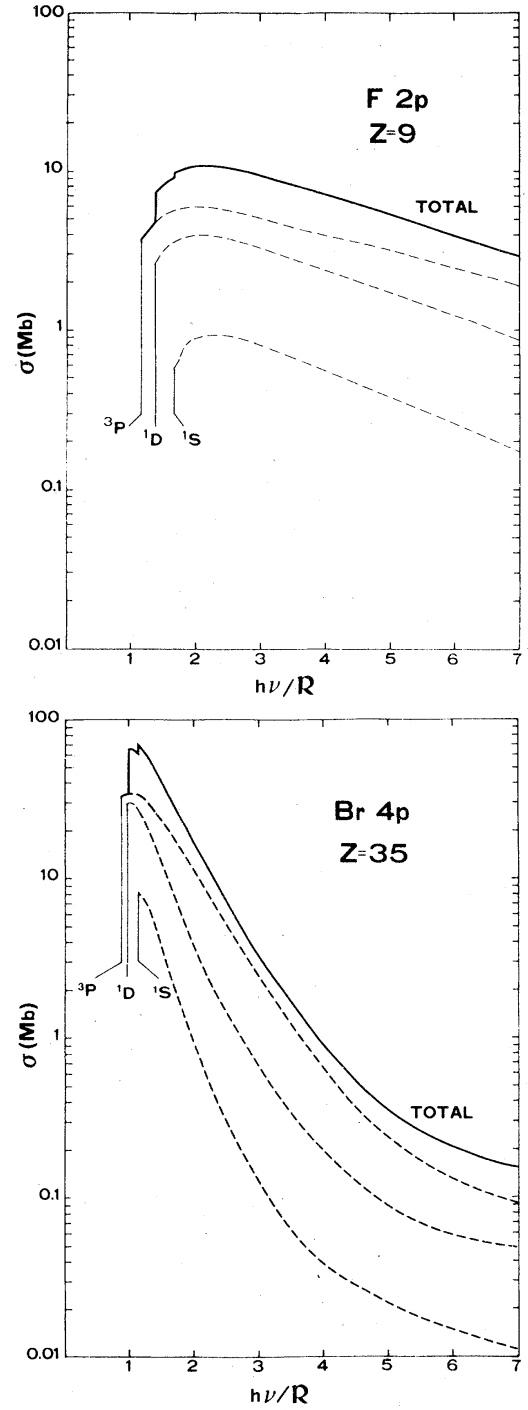
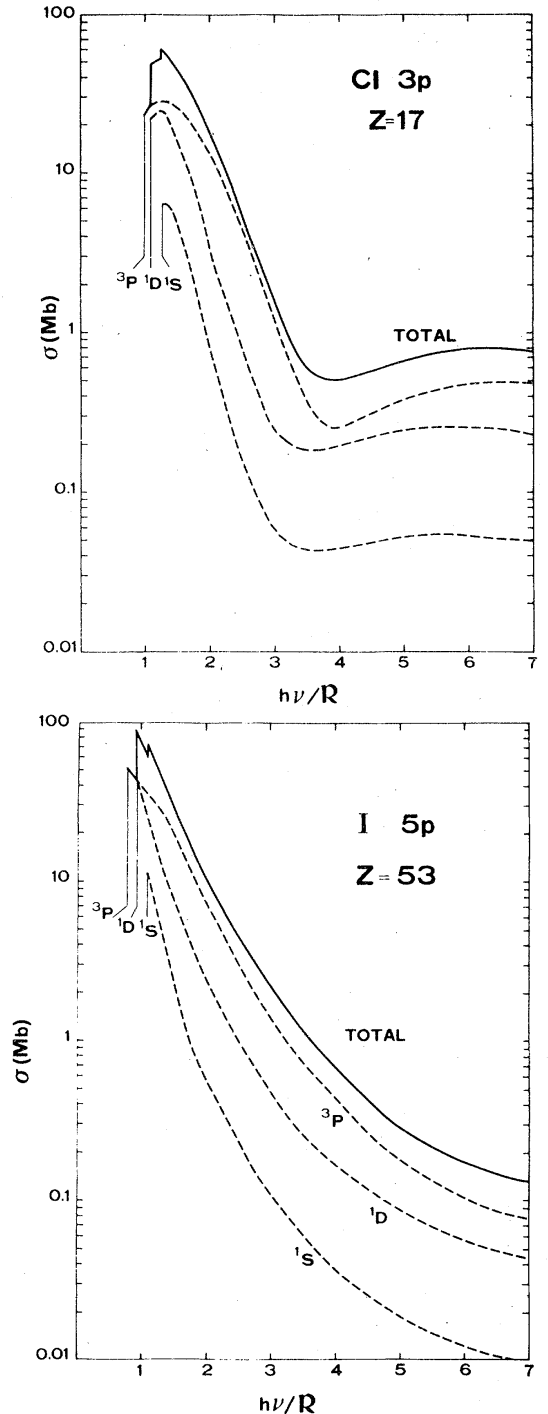

FIG. 1. Photoionization cross sections for the outer $n p$ subshell of the halogen atoms. The partial cross sections for the transitions $n p^{5}\left({ }^{2} P\right)$ $+\omega \rightarrow n p^{4}\left({ }^{3} P,{ }^{1} D,{ }^{1} S\right)+e^{-}$ are indicated by the dashed curves. The total cross section is indicated by the solid curve. levels of the residual ion. The multiplet structure of the final ionic state is seen in the various discrete "jumps" made by the total cross section near each partial cross section threshold. Aside from this threshold structure, however, the halogen and chalcogen atoms in each row of the Periodic Table have total cross sections which are remarkably similar to the noble gas at the end of that row, ${ }^{7}$ as well as to each other.

The cross sections for $O$ and $F$ are quite like each other and each is like that for Ne. These cross sections have no Cooper minima ${ }^{7,49,50}$ since the $2 p$ subshell is nodeless. ${ }^{49}$ The partial cross sections rise slowly above their thresholds, and the total cross section reaches a maximum of $\sim 10 \mathrm{Mb}$ just above threshold. Both partial and total cross sections fall off gently at higher photon energies. The maximum value of the total cross section decreases with increasing $Z$ along a row, i.e., from $\mathrm{O}$ to $\mathrm{F}$ to $\mathrm{Ne}$.

For the $n=3$ row, i.e., $\mathrm{S}$ and $\mathrm{Cl}$, the general shape of the cross section is quite different. Here a Cooper minimum is seen in each partial cross section as well as in the total cross section. The overall shape of each total cross section, away from the multiplet structure near threshold, is very much like that for the Ar $3 p$ subshell. The maximum value attained by the total cross section decreases with increasing $Z$ along a row, i.e., from $\mathrm{S}$ to $\mathrm{Cl}$ to $\mathrm{Ar}$.

The chalcogens and halogens in the next two rows (i.e., Se and $\mathrm{Br}$ for $n=4$ and Te and $\mathrm{I}$ for $n=5)$ all have cross sections which have Cooper minima due to a change in sign of the $n p \rightarrow \epsilon d$ radial dipole matrix element. However, these minima cannot be seen in the figures owing to the 

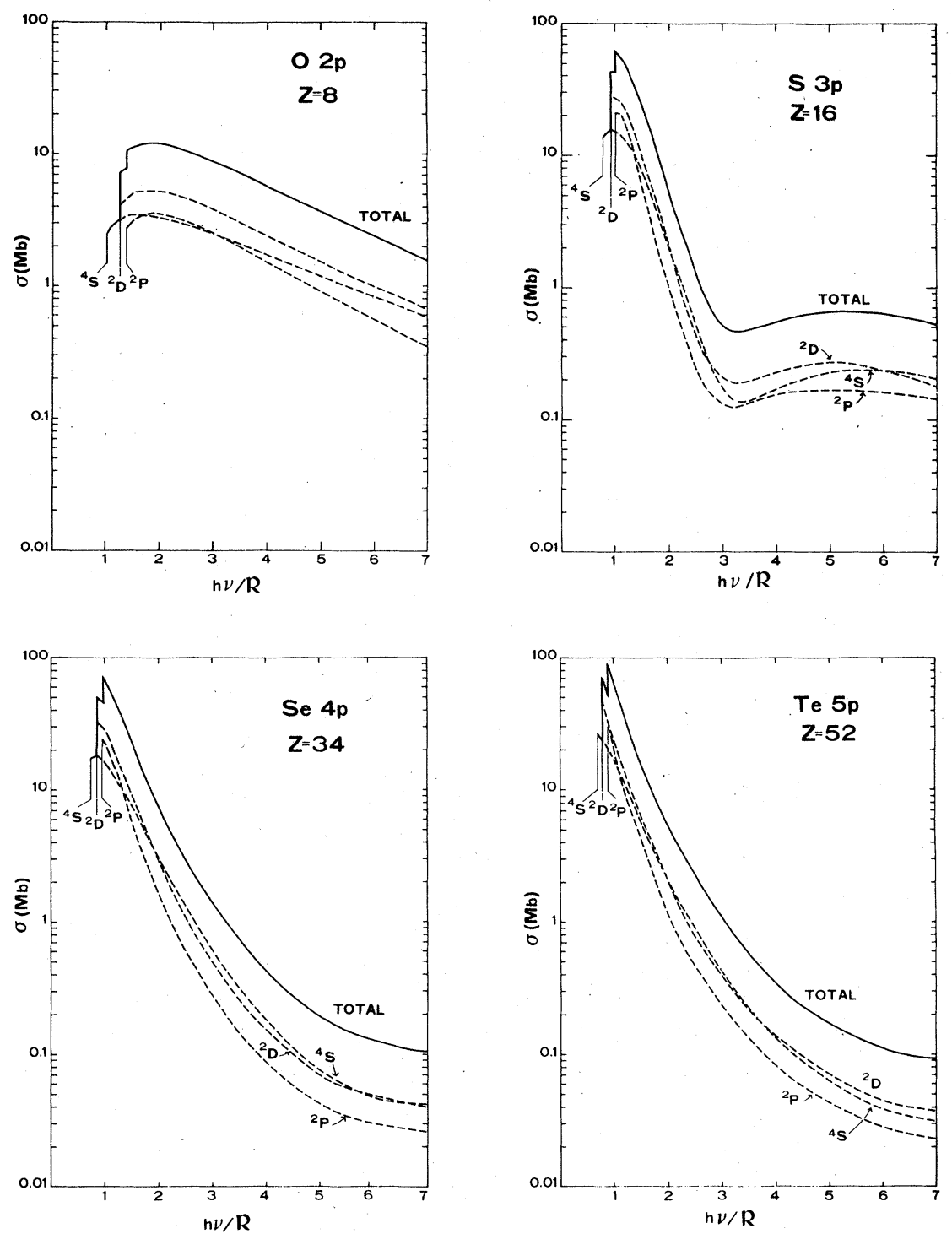

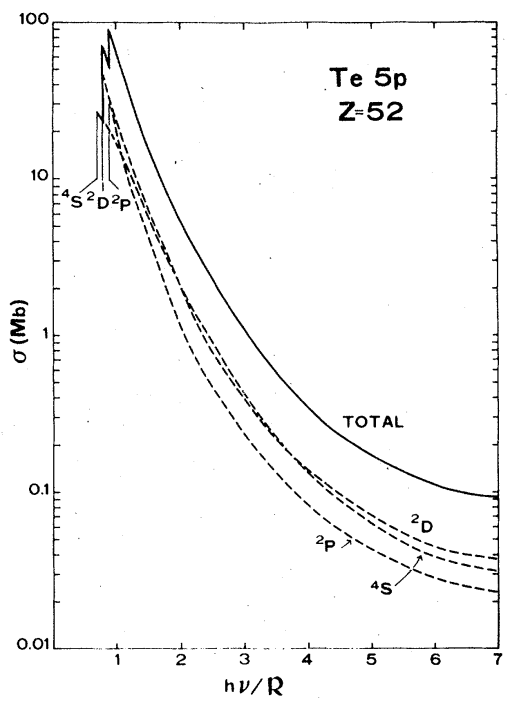

FIG. 2. Photoionization cross sections for the outer $n p$ subshell of the chalcogen atoms. The partial cross sections for the transitions $n p^{4}$ $\left({ }^{3} P\right)+\omega \rightarrow n p^{3}\left({ }^{4} S,{ }^{2} D,{ }^{2} P\right)+e^{-}$ are indicated by the dashed curves. The total cross section is indicated by the solid curve. strength of the $n p \rightarrow \epsilon s$ transition, which dominates the cross section in the neighborhood of the minimum in each case. This corresponds exactly to the same occurrence in the noble gases $\mathrm{Kr}$ and Xe. Even when they do not have a significant effect on the cross sections, these Cooper minima have a very significant effect on the asymmetry parameter $\beta$, as will be discussed in Sec. III B. Aside from this submersion of the Cooper minima, the cross sections for the $n=4$ and $n=5$ subshells behave substantially like those for the $n=3$ row.

Thus the cross sections for the outer subshells of the chalcogens and halogens may be classified according to two distinct patterns, one corresponding to that for the $n=2$ row and the other corresponding to that for the $n=3,4$, and 5 rows with the proviso that the Cooper minimum is clear- ly observable for $n=3$ but submerged for $n=4$ and 5 as noted above. A similar distinction may be made between the cross sections for $\mathrm{Ne}$ and the other rare gases. In fact, each of the rare gases have cross sections similar to that for the chalcogen and halogen atoms in the same row.

One final general feature of the total cross sections is that in going down a column of the Periodic Table one finds the maximum value attained by the total cross section increases while at the same time the total cross section falls off more rapidly at energies above the maximum. This behavior is also exhibited by the noble gases.

Turning now to the individual partial cross sections some general statements can be made. If the term dependence of the ionization thresholds and of the radial dipole matrix elements is neglected 
then the partial cross sections for leaving the halogen ions in the ${ }^{3} P,{ }^{1} D,{ }^{1} S$ term levels have a 9:5:1 branching ratio while the partial cross sections for leaving the chalcogen ions in the ${ }^{2} D,{ }^{4} S$, ${ }^{2} P$ term levels have a 5:4:3 branching ratio. These ratios are obtained by evaluating the geometrical factors in Eqs. (3). ${ }^{51}$ Deviations of our calculated branching ratios from these geometrical ratios are due to dynamical effects which induce a term dependence in the dipole matrix elements and in the ionization energies. It is expected that these term-dependent effects will be of greatest significance near the thresholds where the term-dependent splittings and exchange interactions are most important.

For the halogens, $\sigma\left({ }^{3} P\right)>\sigma\left({ }^{1} D\right)>\sigma\left({ }^{1} S\right)$ over the entire energy range shown in Fig. 1; this is just the ordering implied by the geometrical factors discussed above. Note, however, that the geometrical ratios are fairly large for the halogens so that even near threshold, where the dynamical effects are of greatest importance, they are nevertheless not large enough to change the relative ordering. The magnitudes of the branching ratios are certainly not however, predicted correctly by the geometical ratios near threshold where, e.g., the $\sigma\left({ }^{3} P\right): \sigma\left({ }^{1} D\right)$ ratio for $\mathrm{I}$ is only slightly larger than unity and most assuredly not 9:5.

For the chalogens, shown in Fig. 2, the geometrical ratios do not even predict the relative ordering of the partial cross sections correctly. In fact, there is much crossing of the partial cross sections. This behavior is due to the fact that the geometrical ratios are so close to unity in this case, i.e., 5:4:3, that the dynamical variations of the dipole matrix elements easily cause a reordering of the partial cross sections. At the high-energy end of the curves shown, i.e., for $\omega \simeq 7 \mathrm{Ry}$, the ordering is however as predicted by the geometrical ratios. The measurement of these branching ratios provides important information about dynamical effects in the photoionization process.

The accuracy of the present calculations can best be judged by comparison with experimental data, which are unavailable, however, except for O. A comparison of the present calculation for $\mathrm{O}$ with the experimental data of Cairns and Samson ${ }^{52}$ and Kohl et al. ${ }^{53}$ is shown in Fig. 3. It is seen that above the ${ }^{2} P$ threshold agreement between theory and experiment is good, the theoretical values lying well within experimental error bars. Below the ${ }^{2} P$ threshold, however, the theoretical values are consistently below the experimental measurements. It is believed that this is due to the neglect of autoionizing resonances in the present HF calculations. The energy region between the ${ }^{4} S$ and ${ }^{2} P$ threshold has many autoionizing states so that the measured cross sections should be somewhat higher than our predicted values. This is also consistent with the detailed results of Taylor and Burke $^{19}$ and of Pradhan ${ }^{20}$ which show numerous resonances containing substantial oscillator strength in the region of $15-20 \mathrm{eV}$. This points out an important caveat to bear in mind concerning

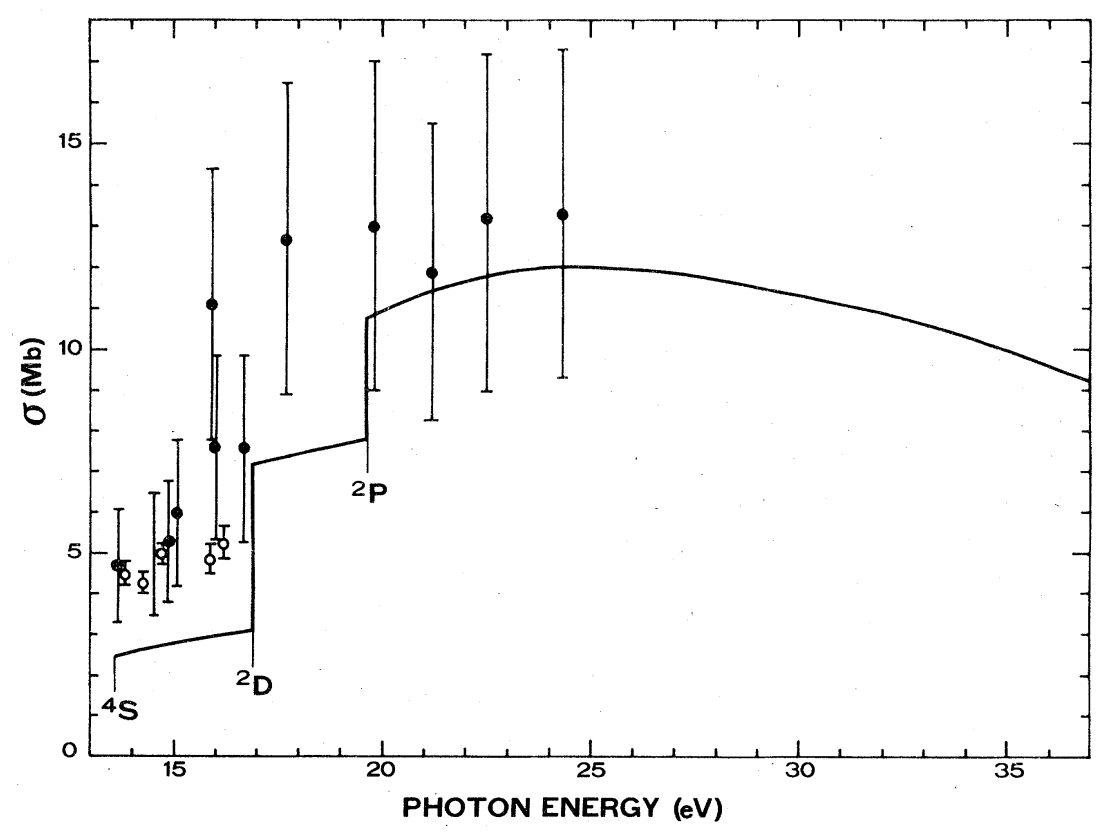

FIG. 3. Photoionization cross section of atomic oxygen. Solid line, present $\mathrm{HF}$ results; solid circles, experimental measurements of Cairns and Samson (Ref. 52); open circles, experimental measurements of Kohl et al. (Ref. 53). 


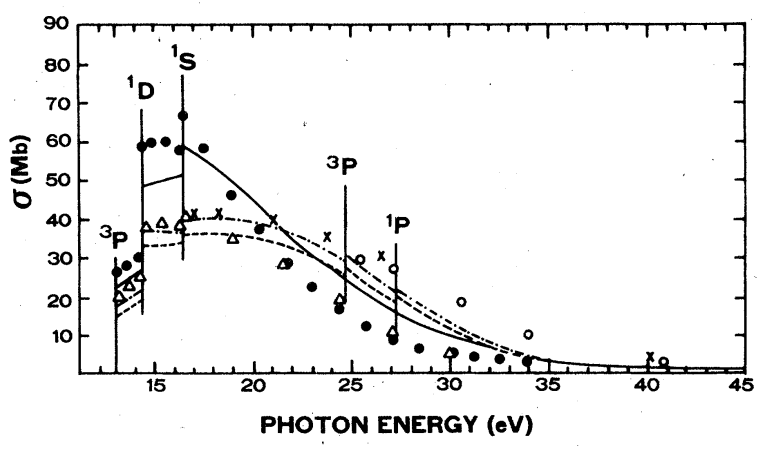

FIG. 4. Theoretical predictions for the photoionization cross section of atomic chlorine. Solid line, present HF results; crosses, close-coupling results of Conneely et al. (Ref. 21); solid circles, RPA results of Starace and Armstrong (Ref. 24); triangles, RPA results of Cherepkov and Chernysheva (Ref. 25); open circles, $R$-matrix results of Lamoureux and CombetFarnoux (Ref. 26); dash-dotted and dashed lines, MBPT length and velocity results of Brown et al. (Ref. 27).

the present calculations: since autoionizing resonances were not included in the present calculations, the theoretical photoionization cross sections in the region of a few $\mathrm{eV}$ below each threshold should not necessarily be considered indicative of the experimentally measured values except in an average sense. Away from the resonances, on the other hand, we find good agreement (within $\sim 10 \%$ ) with Refs. 19 and 20.

Although no experimental cross sections have been reported for the other atoms considered in this paper, numerous more detailed calculations of the photoionization of chlorine have been performed. ${ }^{21,24-27}$ A comparison of our results with these other theoretical results is shown in Fig. 4 . None of the results shown include autoionization so they are directly comparable. It is difficult to reach a conclusive assessment of the accuracy of our results for $\mathrm{Cl}$, as can be seen from Fig. 4, owing to the significant differences which exist among the various calculations. We can conclude, however, that our results are not grossly inconsistent with other theoretical results.

\section{B. Asymmetry parameters}

Employing the formulation described in Sec. II, computations have been carried out for the asymmetry parameter $\beta$ for photoionization of the outer $p$ subshell in each of the halogens $\mathrm{F}, \mathrm{Cl}, \mathrm{Br}$, and $I$ and the chalcogens $O, S, S e$, and Te. Our results are shown in Figs. 5 and 6 , respectively. For each element, $\beta$ is given for each of the three photoelectron groups corresponding to the three ionic term levels. In addition, the results of Hartiree-Slate $\mathrm{r}^{13,44}$ (HS) central potential calculations of $\beta$ are shown for comparison; only a single curve is given for each element since in the central-potential approximation used the dynamical quantities are independent of the term level structure of the ion. These HS results were calculated using Eq. (16).

Inspection of Figs. 5 and 6 shows that for the
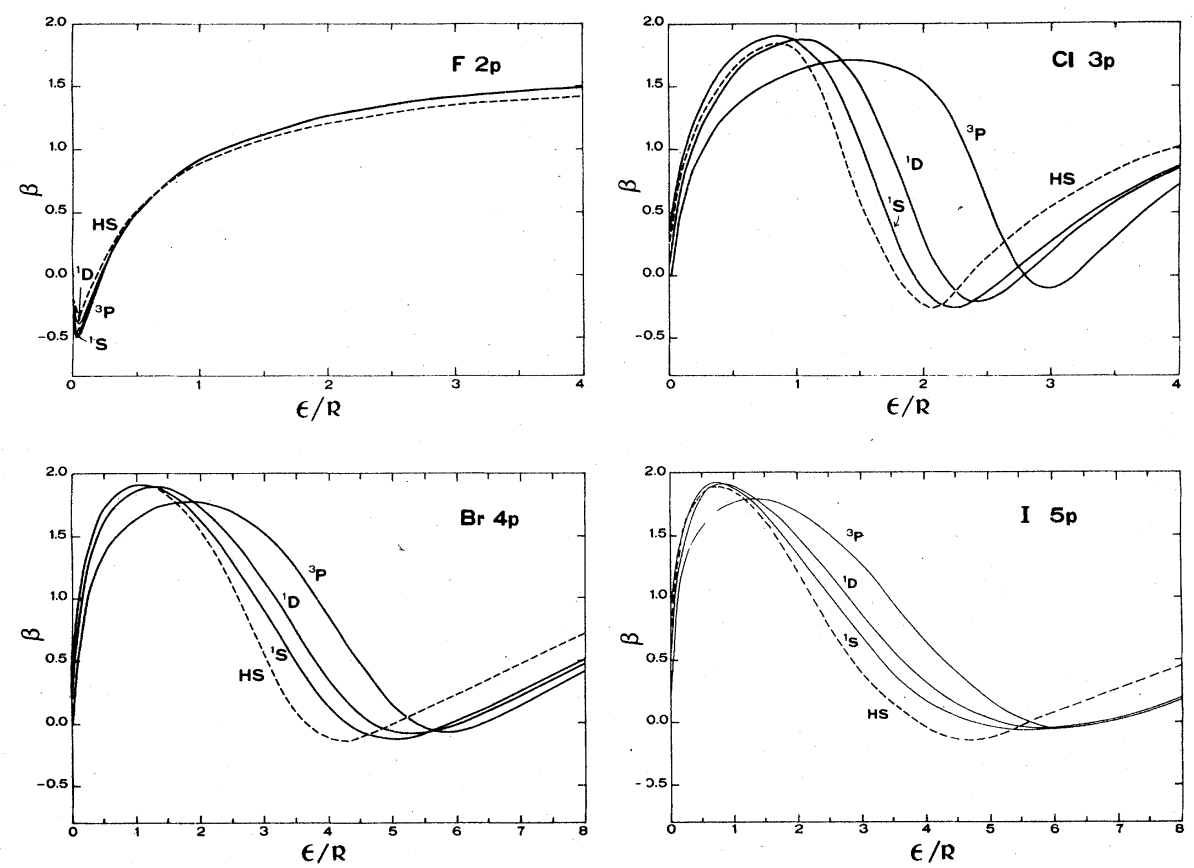

FIG. 5. Asymmetry parameters $\beta$ for the outer $n p^{5}\left({ }^{2} P\right)$ subshells of the halogens. Solid lines, $\beta$ parameters corresponding to the ${ }^{3} P,{ }^{1} D$, and ${ }^{1} S$ ionic terms levels calculated in Hartree-Fock approximation; dashed line, Herman-Skillman central-potential approximation for $\beta$. 

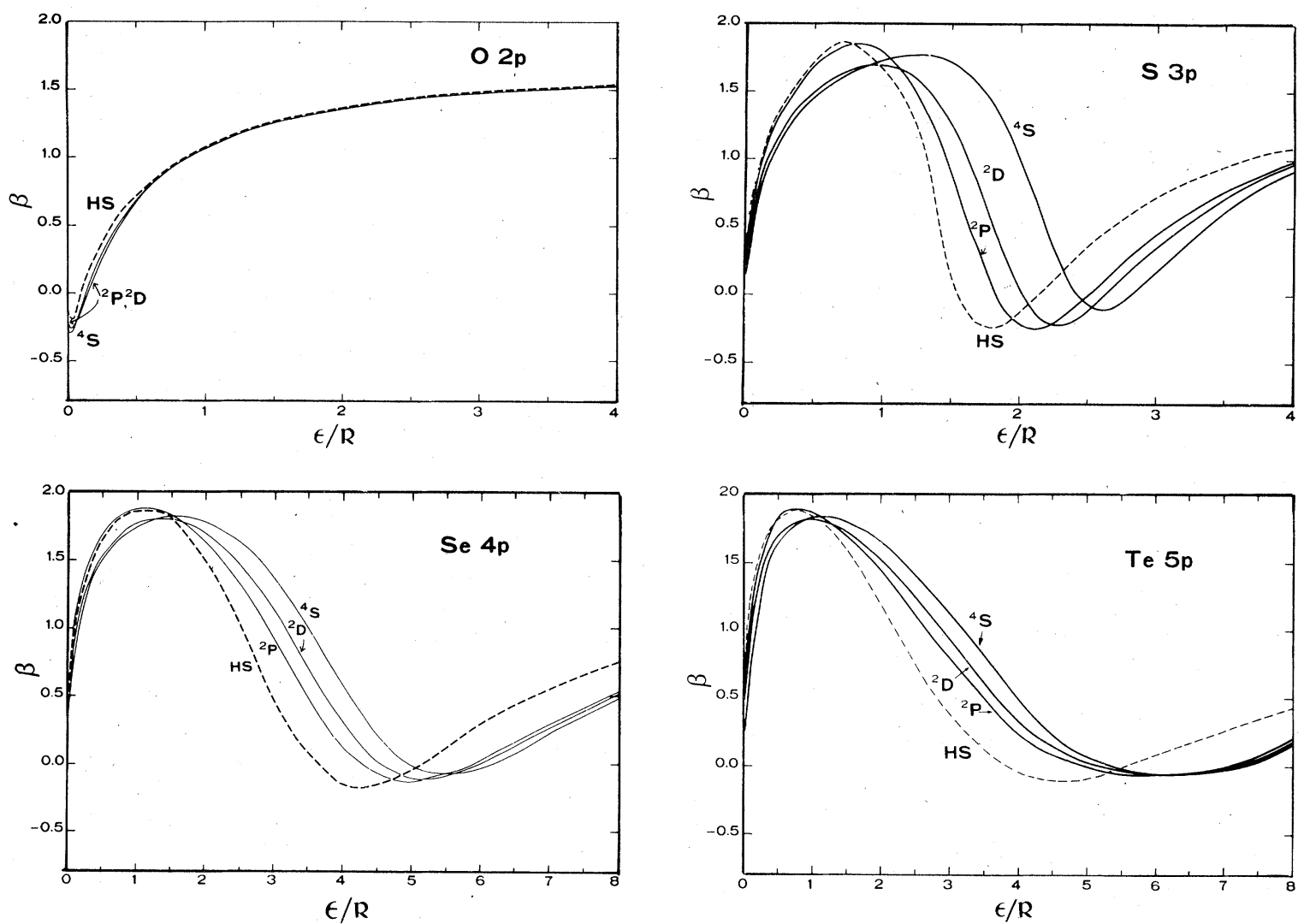

FIG. 6. Asymmetry parameters $\beta$ for the outer $n p^{4}\left({ }^{3} P\right)$ subshells of the chalcogens. Solid lines, $\beta$ parameters corresponding to the ${ }^{4} S,{ }^{2} D$, and ${ }^{2} P$ ionic term levels calculated in Hartree-Fock approximation; dashed line, HermanSkillman central-potential approximation for $\beta$.

halogens and chalcogens the general behavior of the asymmetry parameter $\beta$ as a function of photoelectron energy $\epsilon$ is twofold: one behavior is characterisitic of $p$ subshells whose continuum cross sections have no Cooper minimum, such as those in $\mathrm{F}$ and $\mathrm{O}$; the other is characteristic of $p$ subshells whose continuum cross sections do have Cooper minima, such as those in $\mathrm{Cl}, \mathrm{Br}, \mathrm{I}$, $\mathrm{S}$, Se, and $\mathrm{Te}^{13}$

The asymmetry parameters for $\mathrm{O}$ and $\mathrm{F}$ are very similar and all are similar to that for $\mathrm{Ne}^{7}$ Each $\beta$ has a threshold value that is nearly zero, a short rapid dip and subsequent rise just above threshold, followed by a more gradual rise and a flattening out to a value of about 1.5 at higher photoelectron energies. The HF results for each ionic term level are almost equal to each other and to the HS curve at any given photoelectron energy. This is due to the fact that the exchange interactions of the continuum electron with the ionic core (which are the origin of the term-dependence of dipole matrix elements and phase shifts) are extremely weak in the $n=2$ row of the Periodic Table. The rapid variation of the $\beta$ 's for $O$ and $F$ near threshold is due almost entirely to the rapid variation of the Coulomb phase-shift difference between the $d$ - and $s$-continuum states and is a general characterisitic of the threshold behavior of $p$-subshell asymmetry parameters. ${ }^{13}$

For the chalcogen and halogen atoms in the $n=3$ row of the Periodic Table, i.e., $\mathrm{S}$ and $\mathrm{Cl}$, the other general shape is seen. For these atoms, the $\beta$ 's have a small value near threshold, rise rapidly to a maximum (with a value nearly equal to 2 ), then fall to negative values, and subsequently rise smoothly. This general shape is characteristic of a $p$ subshell whose photoionization cross section has a Cooper minimum. Again, the rapid variation of the $\beta$ 's near threshold is due to the rapid variation of the Coulomb phase shift differences. The sharp drop of the $\beta$ 's near $\epsilon=2 \mathrm{Ry}$ is due to the zeros in the dipole matrix elements which are associated with the Cooper minima in the corresponding cross sections, i.e., the $n p \rightarrow \epsilon d$ dipole matrix elements go through a change of sign in this energy region. The most striking feature of these $n=3$ results, however, is the quantitative difference between the $\beta$ 's for the three ionic term 
levels. This is in sharp contrast to the situation for $\mathrm{O}$ and $\mathrm{F}$ and is due to the much greater strength of the final-state exchange interactions in $\mathrm{S}$ and $\mathrm{Cl}$.

The energy at which $\beta=0$ for a given ion term level corresponds roughly to the energy at which the partial cross section for that ionic term level has a Cooper minimum. This correspondence is exact within the central-potential model, where for a $p$ subshell the Cooper-Zare formula in Eq. (16) gives

$$
\beta=\frac{2 R_{d}^{2}-4 R_{s} R_{d} \cos \left(\sigma_{d}+\delta_{d}-\sigma_{s}-\delta_{s}\right)}{R_{s}^{2}+2 R_{d}^{2}} .
$$

When the radial dipole matrix element for the $p \rightarrow d$ transition, i.e., $R_{d}$, equals zero, Eq. (21) predicts that $\beta=0$. The more general expression for $\beta$ given by Eqs. (9)-(12) is not so easy to analyze since, as shown in Table $\mathrm{I}$, there are in general several final-state $d$ channels associated with a given state of the ion. The radial dipole matrix elements for these final-state channels will in general go through zero at different energies. Thus the energy at which $\beta=0$ is an "average" of the energies at which each of the radial dipole matrix elements goes through zero and corresponds roughly to the location of the Cooper minimum in the associated partial cross section. Generally, the lower the energy at which $\beta=0$ the more attractive is the effective potential seen by the photoelectron. This is because the continuum $d$ wave must move in toward the origin as the photoelectron energy increases in order to produce the cancellation of positive and negative components of the dipole integrand that result in a zero dipole matrix element.

For Cl Fig。 5 shows that $\beta\left({ }^{1} S\right)$ goes through zero at the lowest photoelectron energy, indicating that the effective potential seen by the photoelectron is most attractive for the ${ }^{1} S$ ion term level. By the same argument, the ${ }^{1} D$ effective potential is less attractive and the ${ }^{3} P$ one is the least attractive. Exactly the same sort of phenomenon is seen for $S$ (cf. Fig. 6) where the ${ }^{2} P$ ion term level has the most attractive effective potential and ${ }^{4} S$ the least. These results are a consequence of the different strengths of the exchange interactions between the photoelectron and the ion for each of the ion term levels. Note that for both $\mathrm{Cl}$ and $\mathrm{S}$, the HS result for $\beta$ goes through zero at an energy lower than that of any of the zeros in the $\beta$ 's calculated using HF wave functions. This is because the approximation to exchange in HS is purely attractive and, compared to those in the more accurate HF calculations, is too attractive. For the $n=4$ and $n=5$ rows of the Periodic Table, the situation is substantially similar to $n=3$ described above. The ordering of the zeros in the $\beta$ 's for the different ionic term levels is exactly the same as in the $n=3$ cases and the HS results again indicate the attractive nature of the HS exchange. The ordering remains the same because the relative attractiveness of the exchange interactions is due entirely to geometrical factors, i.e., the geometrical coefficients which enter the HF equations, Eqs. (17). The size of the separations between the $\beta$ 's for different term levels is a dynamical effect which depends upon the detailed shapes of the continuum wave functions and their overlap with discrete ionic orbitals, i.e., it depends upon the magnitude of the exchange integrals.

Figures 5 and 6 show that in going down the columns of the Periodic Table associated with the chalcogens and halogens, there is virtually no separation between the different $\beta$ 's for $n=2$, large separations for $n=3$, and decreased separations for $n=4$ and $n=5$. These separations correspond to the magnitude of the effective photoelectron-ion exchange interactions. The dominant exchange interaction between the $\epsilon d$ electron and the ionic electrons is that with the outer $n p$ subshell. That this interaction is small for the $n=2$ row (i.e., for $\mathrm{O}$ and $\mathrm{F}$ ) is reasonable since the $\epsilon d$ wave function has its first maximum at a rather large radial distance while the nodeless $2 p$ wave function is quite compact. For $n=3$ (i.e., for $\mathrm{S}$ and $\mathrm{Cl}$ ), on the other hand, the $3 p$ wave function is much more diffuse and has a node, so that the overlap with the $\epsilon d$ wave-function is greater and the exchange integrals much larger. Going to the $n=4$ row (i.e., Se and $\mathrm{Br}$ ), the situation is substantially the same as for $n=3$; the $4 p$ and $\epsilon d$ wave functions have one more node each, however, and this leads to some additional cancellation which decreases the exchange slightly from that for $n=3$. This same trend continues to $n=5$ (i.e., Te and I) for precisely the same reasons.

A more quantitative description of these anisotropic exchange interaction effects may be given by introducing certain average phase shifts and radial dipole matrix elements and then considering the magnitude of the deviations about these average values. We introduce first the average phase shift $\bar{\delta}_{\epsilon d}\left(L_{c} S_{c}\right)$ and radial dipole matrix element $\bar{R}_{\epsilon d}\left(L_{c} S_{c}\right)$ at photoelectron energy $\epsilon$ corresponding to the ${ }^{2 S} c^{+1} L_{c}$ ion term level, where these average quantities are defined by the equation

$$
\begin{aligned}
& \exp \left[i \bar{\delta}_{\epsilon d}\left(L_{c} S_{c}\right)\right] \bar{R}_{\epsilon d}\left(L_{c} S_{c}\right) \\
& \equiv\left[l_{0}\right] \sum_{L} \exp \left(i \delta_{\epsilon i}^{L} S_{c} S_{c}\right) R_{\epsilon i}^{L_{c} S_{c} L}[L] \\
& \times\left\{\begin{array}{ccc}
L_{0} & L_{c} & j_{t} \\
2 & 1 & L
\end{array}\right\}\left\{\begin{array}{ccc}
L_{0} & L_{c} & l_{0} \\
2 & 1 & L
\end{array}\right\} .
\end{aligned}
$$


The summation on the right-hand side appears in Eq. (12) for the amplitude $S_{l=2}\left(j_{t}=1\right)$. In the central-potential approximation the phase shifts and dipole matrix elements on the right-hand side of Eq. (22) do not depend on the term level quantum numbers and the summation over angular factors may be performed analytically to yield

$$
\left[l_{0}\right] \sum_{L}[L]\left\{\begin{array}{ccc}
L_{0} & L_{c} & j_{t} \\
2 & 1 & L
\end{array}\right\}\left\{\begin{array}{ccc}
L_{0} & L_{c} & l_{0} \\
2 & 1 & L
\end{array}\right\}=\delta_{j_{t^{1}}} .
$$

Thus in the central-potential approximation Eq. (22) becomes an identity provided that we set $j_{t}=$ $l_{0}=1$ on the right-hand side in Eq. (22). (Note that $j_{t}=1$ is the only angular momentum transfer that contributes to the $p$-subshell cross section and angular distribution asymmetry parameter in the central potential approximation [cf. Sec. II, especially Eq. (14)ff].) Given the average phase shifts and radial dipole matrix elements for each ionic term level according to Eq. (22) (with $j_{t}=1$ ) we now define phase shifts $\hat{\delta}_{\epsilon d}$ and radial dipole matrix elements $\hat{R}_{\epsilon_{d}}$ averaged over the three ionic term levels occurring for the halogens and chalcogens:

$\exp \left(i \hat{\delta}_{\epsilon d}\right) \hat{R}_{\epsilon d} \equiv \frac{1}{3} \sum_{L_{c}, S_{c}} \exp \left[i \bar{\delta}_{\epsilon d}\left(L_{c} S_{c}\right)\right] \bar{R}_{\epsilon d}\left(L_{c} S_{c}\right)$.

Multiply Eq. (24) by $\left[\exp \left(i \delta_{\epsilon d}\right) R_{\epsilon d}\right]^{-1}$ and take the imaginary part of both sides to get

$$
0=\frac{i}{3} \sum_{L_{c}, S_{c}} \frac{\sin \left(\bar{\delta}_{\epsilon d}\left(L_{c} S_{c}\right)-\hat{\delta}_{\epsilon d}\right) \bar{R}_{\epsilon d}\left(L_{c} S_{c}\right)}{\hat{R}_{\epsilon d}} .
$$

The imaginary part on the right-hand side of Eq. (25) must be zero. A measure of the variation of $\bar{\delta}_{\epsilon d}\left(L_{c} S_{c}\right)$ and $\bar{R}_{\epsilon d}\left(L_{c} S_{c}\right)$ about the average values $\hat{\delta}_{\epsilon d}$ and $\hat{R}_{\epsilon d}$ is thus the square root of one-third the sum of the squares of the individual terms in Eq. (25), i.e.,

$$
\overline{\Delta S} \equiv\left(\frac{1}{3} \sum_{L_{c}, S_{c}^{\prime}} \frac{\sin ^{2}\left[\bar{\delta}_{\epsilon d}\left(L_{c} S_{c}\right)-\hat{\delta}_{\epsilon d}\right] \bar{R}_{\epsilon d}^{2}\left(L_{c} S_{c}\right)}{\hat{R}_{\epsilon d}^{2}}\right)^{1 / 2} \geqslant 0 .
$$

Note that in the absence of anisotropic interactions the phase shifts and dipole matrix elements have no term dependence and hence $\overline{\Delta S}=0 . \overline{\Delta S}$ may be correlated with the standard deviation of the asymmetry parameters,

$$
\Delta \beta=\left(\frac{1}{3} \sum_{L_{c}, S_{c}}\left[\bar{\beta}-\beta\left(L_{c}, S_{c}\right)\right]^{2}\right)^{1 / 2},
$$

where $\bar{\beta}$ is the average of $\beta . \overline{\Delta S}$ and $\Delta \beta$ were calculated at each photoelectron energy $\epsilon$ for each element. In Fig. 7 we show a plot of the logarithm of $\Delta \beta$ vs $\overline{\Delta S}$ for a photoelectron energy $\epsilon$ corresponding to the first maximum in $\Delta \beta$ above threshold in each element. We see firstly that $\mathrm{O}$ and $\mathrm{F}$

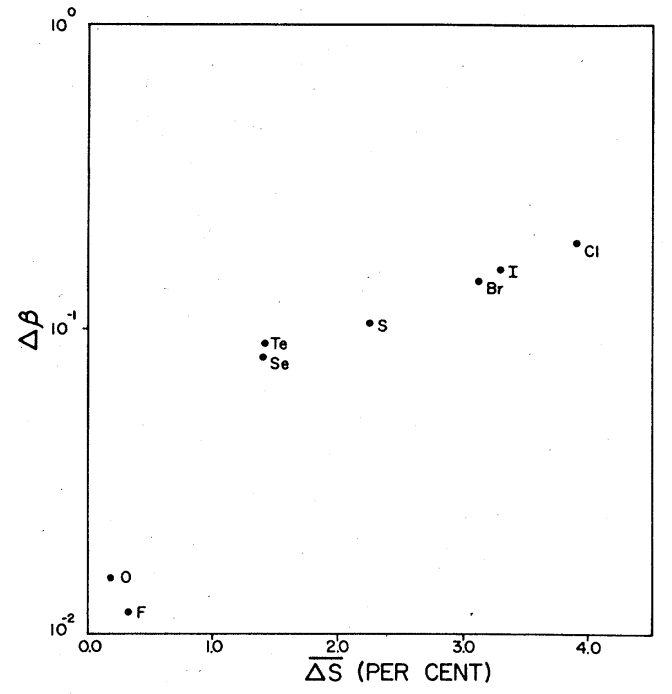

FIG. 7. Plot of the standard deviation $\Delta_{\beta}$ of the three $p$ subshell $\beta$ 's for each of the halogens and chalcogens at a photoelectron energy corresponding to the first maximum in $\Delta \beta$ plotted vs a measure $\overline{\Delta S}$ [defined by Eq. (26)] of the variation in the phase shifts and radial dipole matrix elements at the same photoelectron energy.

(i.e., the $n=2$ row elements) are anomalous. The $n=3,4$, and 5 row elements however appear to follow a linear trend. For both the halogens and chalcogens the $n=3$ row elements $S$ and $C l$ have the largest values of $\overline{\Delta S}$ and $\Delta \beta$ relative to the corresponding $n=4$ and 5 row elements. Furthermore for $n>2$ all the halogens appear to have larger anisotropic effects than the chalcogens. Figure 7 thus quantifies our earlier discussion of the relation between the magnitude of the electronion exchange interactions (and their effect on the phase shifts and radial dipole matrix elements) and the resulting separations between the various angular distribution asymmetry parameters.

\section{FINAL REMARKS}

The Hartree-Fock results presented survey the systematics of the photoionization cross sections, branching ratios, and photoelectron angular distributions of the outer $p$ subshells of the (openshell) halogen $(\mathrm{F}, \mathrm{Cl}, \mathrm{Br}, \mathrm{I})$ and chalcogen $(\mathrm{O}, \mathrm{S}$, Se, Te) atoms. The most striking feature of these results is that anisotropic interactions, which result from exchange forces between the photoelectron and the ion, are negligible in the $n=2$ row $(\mathrm{O}, \mathrm{F})$ and largest in the $n=3$ row $(\mathrm{S}, \mathrm{Cl})$. The size of these interactions is reflected in the splitting among the $\beta$ 's for alternative final ionic states. Thus, measurements of these $\beta$ 's for open-shell atoms will shed light on the strength 
of anisotropic interactions, and should be vigorously pursued.

Another notable feature is that the branching ratios of the partial cross sections for alternative final ionic states differ markedly from the geometrical values for all of the atoms considered except those in the $n=2$ row (i.e., $\mathrm{O}$ and $\mathrm{F}$ ). These deviations are a manifestation of the dynamical interaction effects within the systems studied. Thus branching ratio measurements would give valuable information on the various interaction strengths and should be given high priority. Note that even relative measurements, such as those performed recently at $584 \AA$ for the halogen atoms, ${ }^{54-56}$ are extremely valuable since they provide these branching ratios. However, it would be preferable to perform such measurements over a wide energy range in order to see the trend of the branching ratio data, especially since it is known that such branching ratios vary rapidly in the vicinity of resonances. ${ }^{57,58}$

Finally, note that these results were obtained neglecting correlation effects (i.e., neglecting those electron-interaction effects not included within the HF approximation). It would be extremely useful to know how correlation affects these results. We are fairly certain the qualitative nature of our results would not change, but the magnitude and systematics of any additional anisotropic interactions resulting from the inclusion of correlation would be of great interest. So also would be a knowledge of precisely which types of correlation are the most important ones in each case.

\section{ACKNOWLEDGMENTS}

We wish to thank Dr. Paul Bagus at the IBMSan Jose Laboratory for providing us with wave functions of the iodine and tellurium ions. We also wish to thank Professor Dan Dill of Boston University for valuable discussions. One of us (STM) wishes to acknowledge the National Science Foundation (under Grant No. PHY76-80123) for research support. Another of us (AFS) wishes to acknowledge the Department of Energy (under Contract No. EY-76-S-02-2892.A002) and the Alfred P. Sloan Foundation for research support.
* Present address: Dept. of Physics and Astronomy, Louisiana State Univ., Baton Rouge, La. 70803.

${ }^{1}$ A. F. Starace, in Handbuch der Physik, edited by W. Mehlhorn (Springer-Verlag, Berlin, to be published) Vol. 31.

${ }^{2}$ J. A. R. Samson, in Ref. 1 .

${ }^{3}$ S. T. Manson, Adv. Electron. Electron Phys. 41, 73 (1976); 44, 1 (1977).

${ }^{4}$ Photoionization and Other Probes of Many-Electron Interactions, edited by $\mathrm{F}$. Wuilleumier (Plenum, New York, 1976).

${ }^{5}$ R. D. Hudson and L. J. Kieffer, At. Data 2, 205 (1971).

${ }^{6}$ U. Fano and J. W. Cooper, Rev. Mod. Phys. 40, 441 (1968).

${ }^{7}$ D. J. Kennedy and S. T. Manson, Phys. Rev. A $\underline{5}, 227$ (1972).

${ }^{8}$ E. J. McGuire, Phys. Rev. 175, 20 (1968).

${ }^{9}$ G. M. Thomas and T. M. Helliwell, J. Quant. Spectrosc. Radiat. Transfer 10, 423 (1970).

${ }^{10}$ H. Kähler, J. Quant. Spectrosc. Radiat. Transfer 11, 1521 (1971).

${ }^{11}$ J. U. Koppel, J. Chem. Phys. 55, 123 (1971).

${ }^{12}$ P. S. Ganas, Phys. Rev. A 7, 928 (1973).

${ }^{13}$ S. T. Manson, J. Electron Spectrosc. Relat. Phenom. 1, 413 (1973); 2, 206 (1973); 2, 482 (1973).

${ }^{14}$ A. Dalgarno, R. J. W. Henry, and A. L. Stewart, Planet. Space Sci. 12, 235 (1964).

${ }^{15}$ R. J. W. Henry, Planet. Space Sci. 15, 1747 (1967); 16, 1503 (1968).

${ }^{16}$ S. T. Manson, D. J. Kennedy, A. F. Starace, and D. Dill, Planet. Space Sci. 22, 1535 (1974).

${ }^{17}$ A. F. Starace, S. T. Manson, and D. J. Kennedy,
Phys. Rev. A 9, 2453 (1974).

${ }^{18}$ E. R. Smith, Phys. Rev. A 13, 1058 (1976).

${ }^{19} \mathrm{~K}$. T. Taylor and P. G. Burke, J. Phys. B 9, L353 (1976).

${ }^{20}$ A. K. Pradhan, J. Phys. B 11, L729 (1978).

${ }^{21}$ M. J. Conneely, K. Smith, and L. Lipsky, J. Phys. B 3,493 (1970).

${ }^{22}$ D. Dill, S. T. Manson, and A. F. Starace, Phys. Rev. Lett. 32, 971 (1974).

${ }^{23}$ D. Dill, A. F. Starace, and S. T. Manson, Phys. Rev. A 11, 1596 (1975).

${ }^{24}$ A. F. Starace and L. Armstrong, Jr., Phys. Rev. A 13, 1850 (1976).

${ }^{25} \mathrm{~N}$. A. Cherepkov and L. V. Chernysheva, Phys. Lett. A 60,103 (1977).

${ }^{26} \mathrm{M}$. Lamoureux and F. Combet-Farnoux, in Abstracts of Papers, International Conference on Vacuum Ultraviolet Radiation Physics, Montpellier, France, 1977 (unpúblished), Vol. I.

${ }^{27}$ E. R. Brown, S. L. Carter, and H. P. Kelly, Phys. Lett. A 66, 290 (1978).

${ }^{28} \mathrm{~A}$ final-state channel is defined to be that set of states having in common the same ion core state [i. e., $\left.n p^{q-1}\left(L_{c} S_{c}\right)\right]$ and the same coupling of the photoelectron to this ion core state (i.e., the same quantum numbers $l$ and $L$ ). Thus the states comprising a channel differ only in the photoelectron's kinetic energy.

${ }^{29} \mathrm{~B}$. W. Shore and D. H. Menzel, Principles of Atomic Spectra (Wiley, New York, 1968), Tables 9.2 and 9.3 .

${ }^{30}$ The symbol $\{a b c\}$ represents unity if $|a-b| \leqslant c \leqslant a+b$ and $a+b+c=$ (an integer $)$, and it represents zero 
otherwise.

${ }^{31}$ M. Rotenberg, R. Bivens, N. Metropolis, and J. K. Wooten, Jr., The 3-j and 6-j Symbols (MIT, Cambridge, Mass., 1959).

${ }^{32}$ C. N. Yang, Phys. Rev. 74, 764 (1948).

${ }^{33} \mathrm{~L}$. Lipsky, in Fifth International Conference on the Physics of Electronic and Atomic Collisions: Abstracts of Papers (Nauka, Leningrad, 1967), pp. 617 and 618 .

${ }^{34}$ V. L. Jacobs, J. Phys. B 5, 2257 (1972).

${ }^{35}$ V. L. Jacobs and P. G. Burke, J. Phys. B $\underline{5}$, L67 (1972).

${ }^{36}$ U. Fano and D. Dill, Phys. Rev. A $\underline{6}, 185$ (1972).

${ }^{37} \mathrm{D}$. Dill and U. Fano, Phys. Rev. Lett. 29, 1203 (1972).

${ }^{38}$ D. Dill, Phys. Rev. A 7, 1976 (1973).

${ }^{39} \mathrm{~J}$. Cooper and R. N. Zare, in Lectures in Theoretical Physics, edited by S. Geltman, K. T. Mahanthappa, and W. E. Britten (Gordon and Breach, New York, 1969), Vol. XI-C, pp. 317-337.

${ }^{40} \mathrm{E}$. Clementi and C. Roetti, At. Data Nucl. Data Tables 14, 177 (1974).

${ }^{41} \mathrm{P}$. Bagus (private communication).

${ }^{42}$ A. Dalgarno, R. J. W. Henry, and A. L. Stewart, Planet. Space Sci. 12, 235 (1964).

${ }^{43} \mathrm{~J}$. C. Slater, Quantum Theory of Atomic Structure (McGraw-Hill, New York, 1960), Vol. II, pp. 286-291.

${ }^{44} \mathrm{~F}$. Herman and S. Skillman, Atomic Structure Cal- culations (Prentice-Hall, Englewood Cliffs, N. J. 1963).

${ }^{45}$ A. F. Starace, Phys. Rev. A 3, 1242 (1971); 8, 1141 (1973).

${ }^{46}$ I. P. Grant and A. F. Starace, J. Phys. B 8, 1999 (1975).

${ }^{47}$ A. Hibbert, J. Phys. B 7, 1417 (1974).

${ }^{48}$ S. T. Manson and D. J. Kennedy, Chem. Phys. Lett. 7 , 387 (1970).

${ }^{49} \bar{J}$. W. Cooper, Phys. Rev. 128, 681 (1962).

${ }^{50} \mathrm{~S}$. T. Manson and J. W. Cooper, Phys. Rev. 165, 126 (1968).

${ }^{51} \mathrm{~A} . \mathrm{R} . \mathrm{P}$. Rau, in Electron and Photon Interactions with Atoms, edited by H. Kleinpoppen and M. R. C. McDowell (Plenum, New York, 1976), pp. 141-148.

${ }^{52}$ R. B. Cairns and J. A. R. Samson, Phys. Rev. 139, A1403 (1965).

${ }^{53}$ J. L. Kohl, G. P. Lafyatis, H. P. Palemius, and W. H. Parkinson, Phys. Rev. A 18, 571 (1978).

${ }^{54} \mathrm{D}$. M. de Leeuw, R. Mooyman, and C. A. de Lange, Chem. Phys. Lett. 54, 231 (1978).

${ }^{55} \mathrm{~K}$. Kimura, T. Yamazaki, and Y. Achiba, Chem. Phys. Lett. 58, 104 (1978).

${ }^{56} \mathrm{~J}$. Berkowitz (private communication).

${ }^{57}$ P. C. Kemeny, J. A. R. Samson, and A. F. Starace, J. Phys. B 10, L201 (1977).

${ }^{58}$ A. F. Starace, Phys. Rev. A 16, 231 (1977). 